\title{
ASL and susceptibility-weighted imaging contribution to the management of acute ischaemic stroke
}

\author{
Sébastien Verclytte $\mathrm{e}^{1,2}$ (D) Olivier Fisch ${ }^{1,2} \cdot$ Lucie Colas $^{1,2} \cdot$ Olivier Vanaerde $^{1,2} \cdot$ \\ Manuel Toledano ${ }^{1,2}$ • Jean-François Budzik ${ }^{1,2}$
}

Received: 13 June 2016 /Revised: 19 September 2016 / Accepted: 3 October 2016 / Published online: 7 November 2016

(C) The Author(s) 2016. This article is published with open access at Springerlink.com

\begin{abstract}
Magnetic resonance imaging (MRI) plays a central role in the early diagnosis of cerebral vascular events. Today, MRI is used not only for the detection of acute ischaemic lesions, but also to fine tune the diagnosis and improve patient selection for early therapeutic decisionmaking. In this perspective, new tools such as arterial spin labelling (ASL) and susceptibility-weighted imaging (SWI) sequences have been developed. These MRI sequences enable noninvasive assessment of brain damage, providing important diagnostic and prognostic information: evaluation of cerebral parenchymal perfusion; detection and aetiological assessment of thrombi; ruling out differential diagnoses. After a brief recall of the fundamental basis of these sequences, this article proposes an update on their current contribution to the early management of stroke victims.

\section{Teaching Points}

- These noninvasive sequences provide essential information for early management of acute stroke.

- They can detect zones of parenchymal hypoperfusion.

- Susceptibility-weighted sequences provide information on thrombus localisation and composition.

- ASL can identify certain aetiologies of stroke mimics.

- Post-therapeutic ASL perfusion status predicts outcome.
\end{abstract}

Sébastien Verclytte

verclytte.sebastien@ghicl.net

1 Imaging Department, Lille Catholic Hospitals, Lille, France

2 Lille, France, Lille Catholic University, Lille, France
Keywords Susceptibility-weighted imaging $\cdot$ Arterial spin labelling $\cdot$ Perfusion $\cdot$ Stroke $\cdot$ MRI

\section{Introduction}

MRI is an advanced tool for pre-therapeutic management of acute stroke. MRI can be used to assess the extent of brain infarction, localise the site of arterial occlusion, and search for evidence ruling out potential contraindications for thrombolysis. In recent years, the advent of 3-T MRI scanners for routine clinical applications has incited interest in new sequences exploiting the higher field strength, e.g. arterial spin labelling (ASL) and susceptibility-weighted imaging (SWI) sequences. This opens the way for new perspectives such as noninvasive assessment of parenchymal hypoperfusion, precise localisation of the thrombus and its origin, or characterisation of nonvascular stroke mimics. Here we propose an update on the contribution of these new techniques for acute-phase management of stroke patients.

\section{SWI sequences}

\section{Fundamentals}

These gradient-echo sequences are acquired with a long echo time (TE) in order to take full advantage of the magnetic susceptibility phenomenon. Magnetic susceptibility corresponds to the variation in the local magnetic field of a material exposed to an external magnetic field. This occurs for instance in the venous compartment, which contains a large amount of deoxyhaemoglobin, a highly paramagnetic substance. Paramagnetic substances create a field oriented in the same direction as the higher intensity main field, leading to a lower local 
signal. When a long TE is used, the dephasing resulting from spin-spin interactions and field heterogeneity is increased. TE can thus be set to yield a phase opposition phenomenon between deoxyhaemoglobin and the adjacent parenchyma, further lowering the signal [1]. Moreover, magnetic susceptibility evolves proportionally with the $\mathrm{B} 0$ magnetic field so that the phenomenon is more marked with $3 \mathrm{~T}$ than $1.5 \mathrm{~T}$.

There are several types of susceptibility-weighted sequences. Susceptibility-weighted imaging (Siemens Heathcare, Erlangen, Germany) and Venobold (Philips Healthcare, Best, The Netherlands) are based exclusively on reading a long TE. Other sequences, such as susceptibilityweighted angiography (SWAN) (General Electrics Healthcare, Milwaukee, WI, USA) and susceptibilityweighted imaging with phase enhancement (SWIp) (Philips Healthcare, Best, The Netherlands), are based on reading multiple TEs set at long and short values. This method takes advantage of the more marked time-of-flight (TOF) effect when reading shorter TEs, adding to the magnetic susceptibility effect observed on longer TE images.

\section{Applications for pre-therapeutic management of acute ischaemic stroke}

\section{Haemorrhagic transformation}

Susceptibility-weighted sequences are much more sensitive for the detection of haemorrhagic transformation than either non-contrast CT scan or T2 gradient echo sequences. This greater sensitivity is important not only in the acute phase of ischaemic stroke, but is also highly contributive to the diagnosis of all types of intracranial bleeding [2-5].

\section{Arterial thrombus}

One of the major challenges for MRI exploration of acute stroke is to search for aetiological elements and factors predictive of post-therapeutic outcome. SWI sequences provide information on thrombus localisation and composition.
Intra-arterial signal voids on T2 gradient echo images, termed susceptibility vessel sign, were initially described as suggestive of cardioembolic thrombi [6, 7]. Indeed, those thrombi are mainly composed of red cells and thus blood degradation products have a strong paramagnetic effect compared with fibrin-rich atheromatous thrombi [8].

It appears that a more recently described two-layered susceptibility sign would be more sensitive and much more specific for cardioembolic thrombi than the susceptibility vessel sign (Fig. 1), which can arise via many mechanisms [9].

Whatever the origin of the thrombus, in acute ischaemic stroke, the susceptibility vessel sign would be correlated with:

- a lower rate of recanalisation after intravenous thrombolysis compared with arterial occlusion without the susceptibility vessel sign $[10,11]$, particularly for proximal localisations [12], for lengths greater than $20 \mathrm{~mm}$, for thrombi with irregular contours [13], and for susceptibility artefacts extending beyond the arterial lumen [14];

- a favourable 3-month functional outcome in patients who undergo mechanical thrombectomy for anterior circulation occlusion [15], but not with a higher rate of recanalisation [16].

Because of its greater sensitivity, and particularly so with a strong magnetic field, SWI offers a more precise assessment of thrombus morphology. For determining the site of occlusion, SWI exhibits better sensitivity and specificity than T2 gradient echo (Fig. 2) or 3D TOF imaging [17-19]. It is also much more effective in identifying distal thrombi, for both anterior [17, 20, 21] and posterior [19] localisations (Fig. 3). Detection of multiple distal thrombi is of major importance, since in this situation the 3-month functional outcome is less favourable compared with a unique occlusion [22]. However, distal thrombi may be confused with hypointense venous structures or microbleeds on SWI. The sequences based on a multi-TE readout are more efficient in doubtful cases. Indeed, the TOF effect related to the shortest TE read allows confirming the intra-arterial origin of the signal void assigned to the thrombus thanks to the susceptibility effect.
Fig. 1 An 85-year-old patient presenting left hemibody deficit on DWI (a) and SWI (b and c) sequences in the axial plane. a Acute superficial sylvian and deep right ischaemic event. b and c Long thrombus in the M1 segment of the right middle cerebral artery with a two-layered susceptibility sign

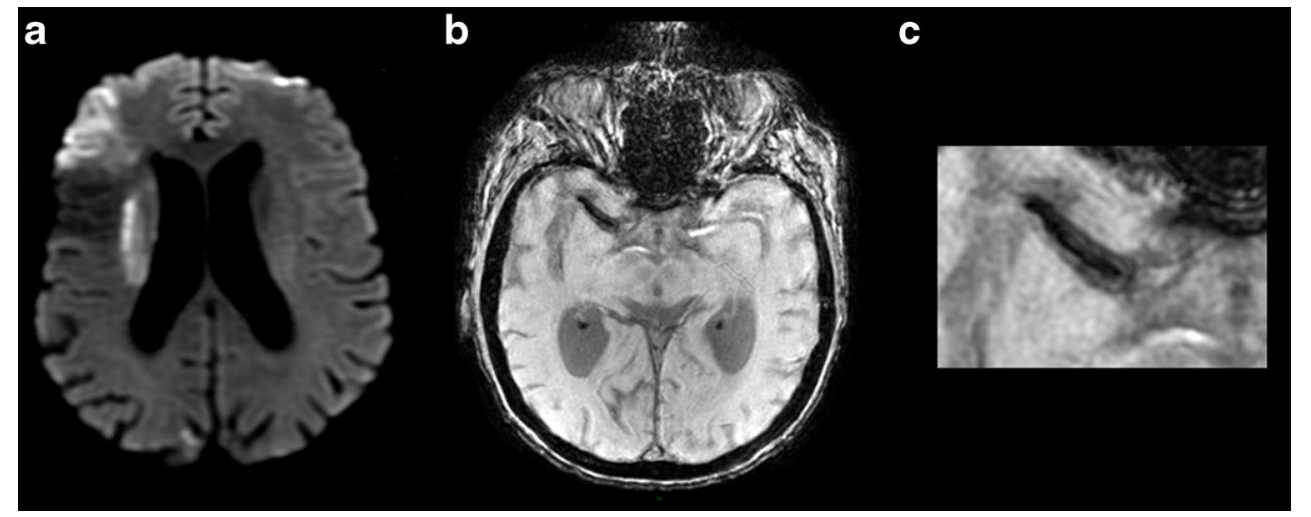




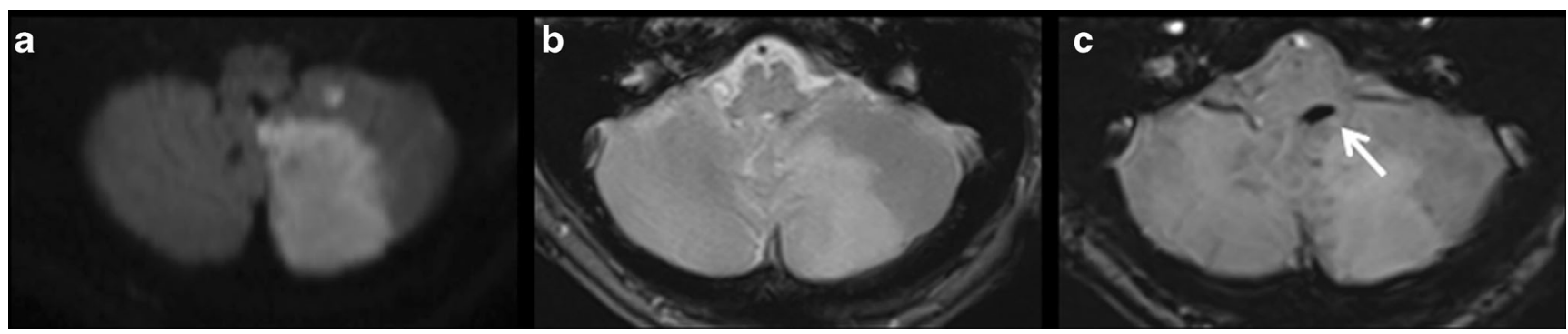

Fig. 2 A 48-year-old patient presenting sudden-onset vertigo. DWI (a), T2 spin-echo (b), and SWI (c) sequences in the axial plane. a Acute ischaemic lesion in the territory of the left posterior inferior cerebellar

\section{Brush sign}

Thanks to the BOLD effect, SWI sequences can also be used to indirectly assess deoxyhaemoglobin content in peri-encephalic veins. Indeed, when exposed to experimental hypoxia, the venous compartment gives a proportionally lower signal that can be detected visually [23]. During acute ischaemia, the local oxygen deprivation secondary to arterial occlusion is seen as a hypointense zone in the cortical and deep veins called the brush sign [24], as multiple hypointense vessels [25], or as prominent vessel [26]. The presence of these signs in the acute phase is associated with a less severe clinical presentation (lower initial NIHSS score), lower lesion diffusion-weighted imaging (DWI) volume, more extensive penumbra, and more pronounced collaterals [25]. Moreover, the brush sign, reflecting cerebral hypoperfusion, would be correlated with penumbra volume. Luo et al. [27] demonstrated the absence of significant mismatch between DWI-MTT (mean transit time) maps produced by the dynamic susceptibility contrast MRI (DSC-MRI) and DWISWI maps (Fig. 4). Susceptibility-weighted sequences would artery. b No intra-vascular signal anomaly. c Susceptibility vessel sign revealing an intra-arterial thrombus in the left posterior inferior cerebellar artery

thus enable effective noninvasive measurement of the penumbra in acute ischaemic stroke.

In the absence of thrombolytic treatment, the initial extent of the brush sign would be correlated with the final infarct volume and the severity of the functional outcome [26]. In case of middle cerebral artery occlusion treated by intravenous thrombolysis, the presence of a brush sign would be associated with a higher risk of haemorrhagic transformation and a less favourable 3-month functional outcome [28].

\section{Arterial spin labelling}

\section{Fundamentals}

Arterial spin labelling (ASL) is a brain perfusion sequence that does not require contrast injection. A salve of radiofrequency waves is applied to a box positioned in the neck area, upstream from the brain region to be studied in order to locally saturate the proton spins of the water molecules in the arterial blood and thus
Fig. 3 A 72-year-old patient with right homonymous lateral hemianopsia. DWI (a), 3D TOF (b), minimum intensity projection (c), and multiplanar reconstruction (d) of the SWAN sequence in the axial plane. a Acute ischaemic lesion in the territory of the left posterior cerebral artery. b No visualisation of the left P2 (white arrow). $\mathbf{c}$ and $\mathbf{d}$ Susceptibility vessel sign in $\mathrm{P} 2$ (curved arrow). d TOF effect of the SWAN sequence identifies the susceptibility vessel sign associated with the thrombus and the upstream arterial segment (arrowhead)

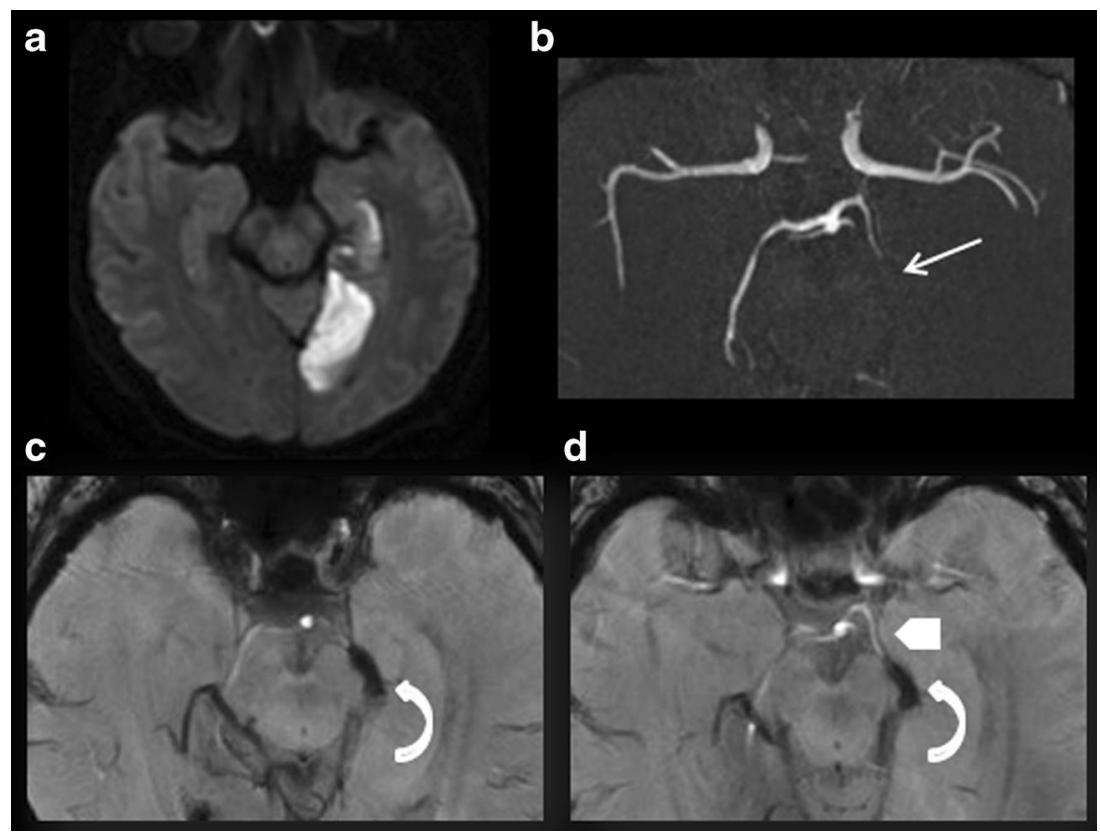


Fig. 4 A 76-year-old patient seen in an emergency setting for right brachiofacial motor deficit $3 \mathrm{~h}$ after symptom onset. DWI (a), FLAIR (b), ASL (c), ASL/DWI fusion (d), and SWI (e and f) sequences in the axial plane. a Acute right superficial sylvian infarction. b The FLAIR sequence fails to visualise any infarct zone. Slow circulation in the cortical branch of the right middle cerebral artery, hypersignal (curved arrow). c Blue zone (white parentheses) visualises a wide right sylvian zone of hypoperfusion. d Mismatch: DWI hypersignal and ASL hypoperfusion. e Susceptibility vessel sign in the M2 segment of the right middle cerebral artery, thrombus. f Right sylvian (white parentheses) brush sign; the extension is the same as the hypoperfusion zone visible on the ASL sequence (image c)

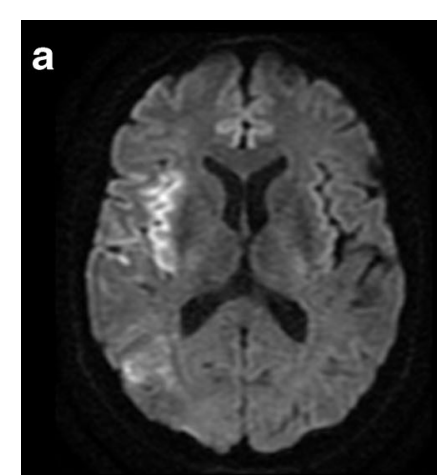

b

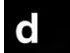

d

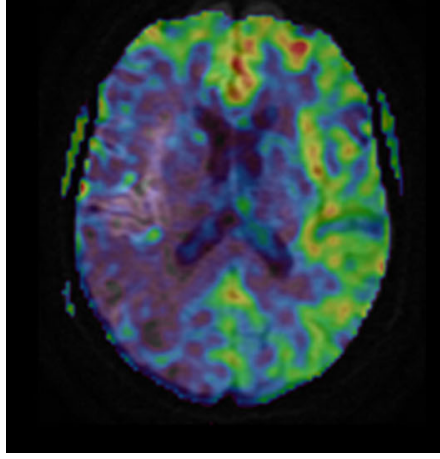

e

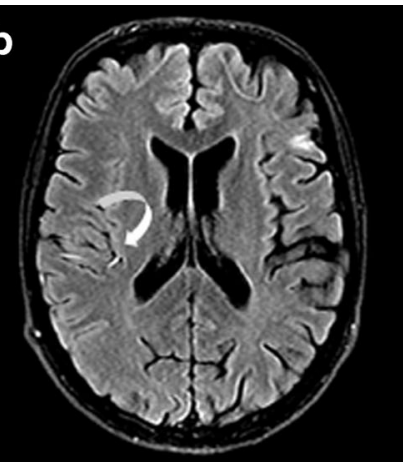

c

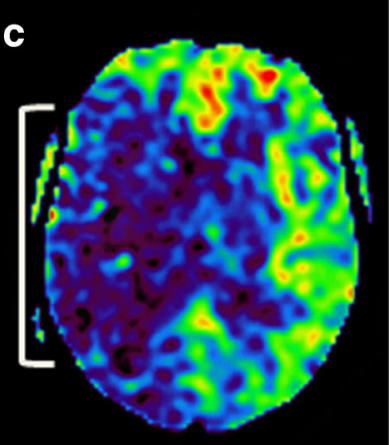

f

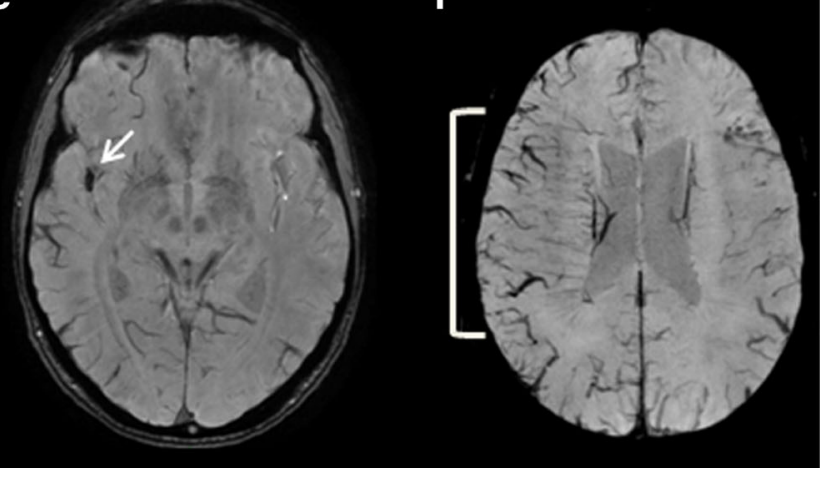

play the role of an endogenous contrast agent. After a certain transit time, which depends upon the subject's age and circulatory conditions, the saturated protons reach the brain parenchyma generating the labelled image. A second acquisition is made without prior saturation of the water molecule spins, generating a control image. Subtraction of the labelled and control images generates a perfusion-weighted image used to produce an absolute measurement of cerebral blood flow (CBF). There are several types of ASL sequences. Continuous ASL (CASL), pulsed ASL (PASL), and pseudo-continuous (pCASL) are based on different excitation methods and present specific advantages and disadvantages.

In general, the CASL method has a higher signal-to-noise $(\mathrm{S} / \mathrm{N})$ ratio but induces an excessive specific absorption rate, particularly at $3 \mathrm{~T}$. With PASL, labelling is particularly effective, but with a low $\mathrm{S} / \mathrm{N}$ ratio. The pCASL method has the advantages of both the preceding methods, with a satisfactory $\mathrm{S} / \mathrm{N}$ ratio and a limited specific absorption rate. It is currently recommended for clinical applications, preferably with turbo spin-echo 3D acquisition [29].

Post-labelling delay (PLD) should to be optimal for pCASL. This parameter should be adjusted on a case-by-case basis and correspond as closely as possible to the time needed for labelled protons to reach the region of interest. If the PLD is too short, all of the labelled bolus may not have time to fully integrate the parenchyma to be explored, particularly junctional areas. This can lead to a significant local signal loss, which could be misdiagnosed as false hypoperfusion regions. Patient-related factors can also lead to systemised false hypoperfusion areas, resulting for example from stenosis of the supra-aortic trunks, which produces a longer transit time between the labelling zone and the region of interest. In this case, it may be difficult to differentiate between false and real hypoperfusion, and other sequences, such as DWI or MR angiography, may be helpful to confirm the diagnosis.

Other artefacts related to the arterial transit can also occur. Seen as linear or serpingious hypersignals within the arteries of the Willis polygon, they are related to the persistence of labelled protons in the vascular compartment because of an overly short PLD. PLD is thus an essential parameter that must be adjusted to the patient's circulatory status.

Standardised PLD values have been validated for patient age and pathological condition: $1500 \mathrm{~ms}$ for children; $1800 \mathrm{~ms}$ for healthy adults aged $<70$ years, and $2000 \mathrm{~ms}$ for adults aged $>70$ years or for patients with a suspected neurological condition, irrespective of the origin [29].

It should be pointed out that ASL is sensitive to motion. It is recommended to use background suppression and prospective correction methods to reduce motion artefacts [29]. However, in cases of highly agitated or confused patients, good quality ASL maps remain difficult to obtain.

\section{Applications for the exploration of acute ischaemic stroke}

\section{Evaluation of the penumbra zone and DWI/perfusion mismatch}

Several 3-T MRI studies have provided objective evidence of the good correlation among computed tomography perfusion, 
Fig. 5 A 76-year-old patient seen in an emergency setting for sudden-onset left hemibody hypoesthesia. DWI (a), ASL (b), 3D TOF (c), and SWI (d) sequences in the axial plane. a Small infarct zone in the right internal temporal region. $\mathbf{b}$ Intravascular hypersignal; bright vessel sign upstream from the thrombus (arrowhead). Right occipital hypoperfusion with DWI mismatch (curved arrow). c Visualisation defect in the $\mathrm{P} 2$ segment of the right posterior cerebral artery (white arrow). d Susceptibility vessel sign in P2; thrombus (black arrow)

Fig. 6 Control image $24 \mathrm{~h}$ after intravenous thrombolysis in a patient seen in an emergency setting for a superficial left sylvian ischaemia with favourable clinical outcome. DWI (a and c) SWI/ASL fusion (b), and ASL (d) sequences. a Left superficial sylvian acute ischaemic lesion. b Zones of hypointense haemorrhagic transformations on the SWI sequence superimpose with the hyperperfusion zones on the ASL (arrowheads). $\mathbf{c}$ and $\mathbf{d}$ Anterior sylvian involvement with partial recovery on the DWI images of the posterior portion corresponding to the zone of hyperperfusion (white arrows)

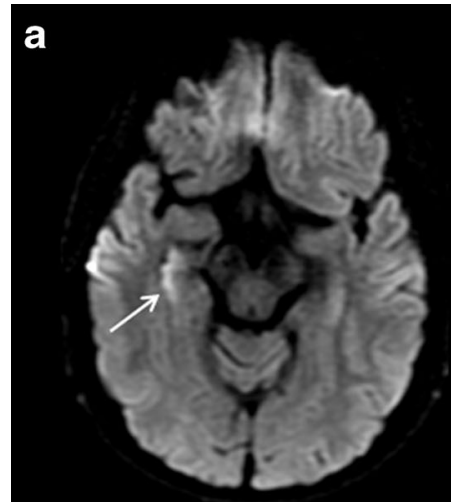

C

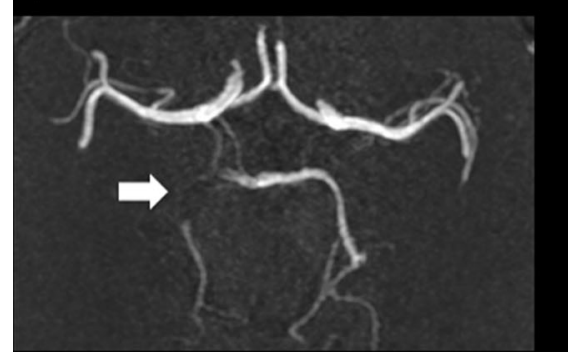

b

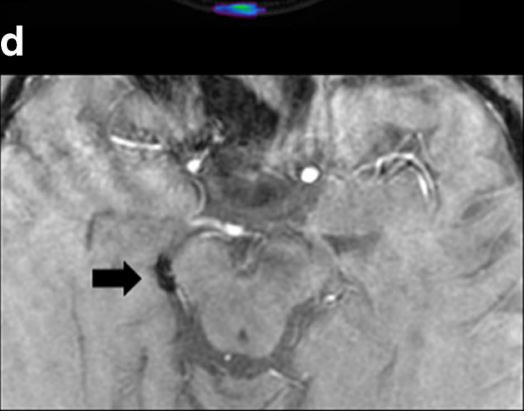

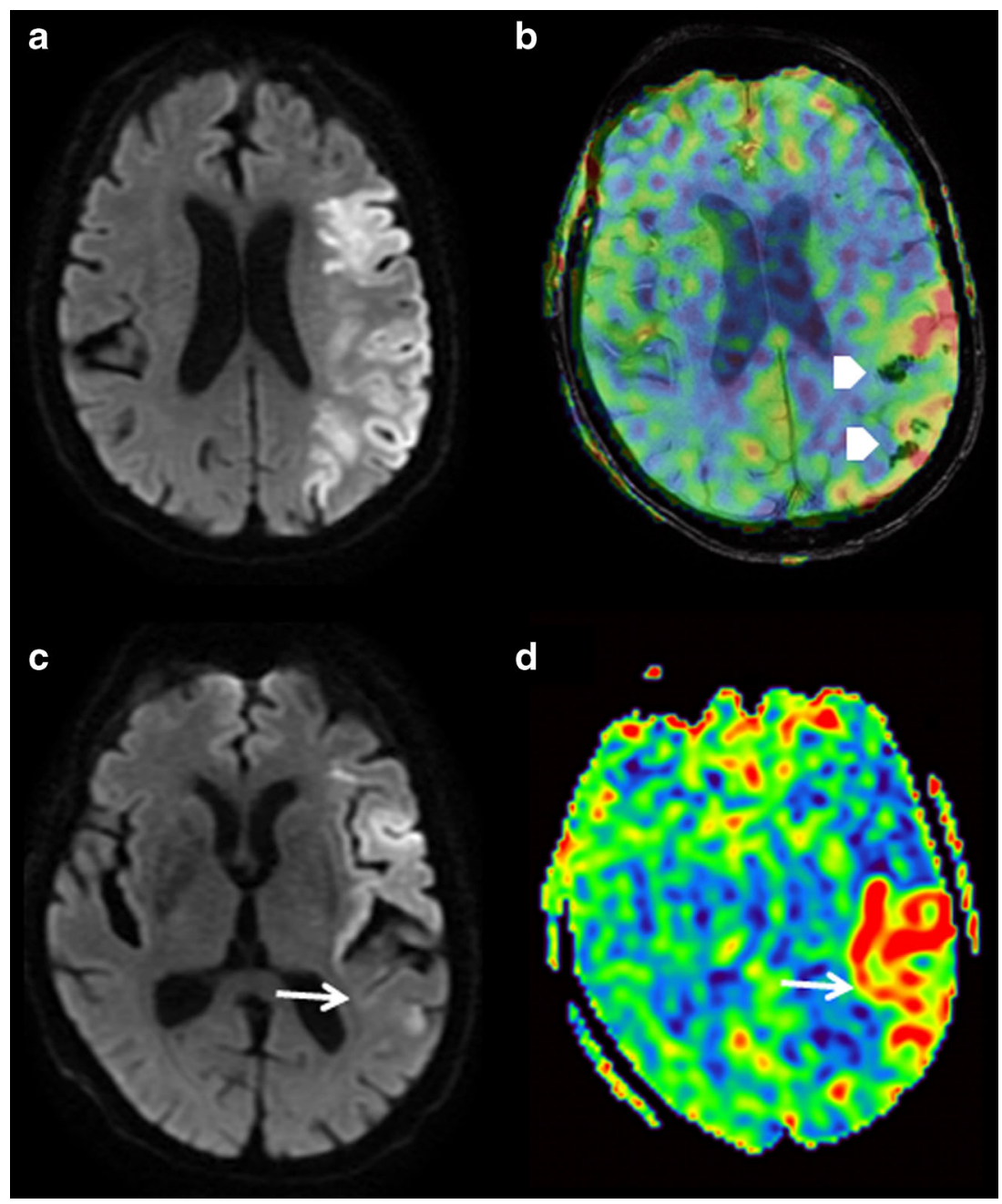


DSC-MRI, and ASL for determining zones of parenchymal hypoperfusion in acute stroke (Fig. 4) [30-33]. Thus ASL provides a reliable assessment of penumbra volume based on the following CBF values:

- ASL-CBF $<20 \mathrm{ml} / 100 \mathrm{~g} / \mathrm{min}$. This is correlated with MTT $>10$ s on DSC-MRI [34];

- $\quad$ ASL-CBF $40 \%$ lower than in healthy contralateral tissue. Lesion volumes thus determined are correlated with volumes measured on computed tomography perfusion maps (Tmax $5.5 \mathrm{~s}$ ) and DSC-MRI (Tmax $+6 \mathrm{~s}$ ) as well as with the 24-h DWI lesion in patients without reperfusion [30].

ASL reliability and reproducibility have been established for 3-T assessment of penumbra volume, while the lower $\mathrm{S} / \mathrm{N}$ ratio hampers $1.5-\mathrm{T}$ performance [35].

\section{Localisation of the arterial thrombus}

During the acute phase of ischaemic stroke, a bright vessel appearance on ASL sequences localises the thrombus (Fig. 5). This bright vessel sign corresponds to an accumulation of protons in labelled arterial blood immediately upstream from the arterial occlusion. The sensitivity of the bright vessel sign would be superior to that of the susceptibility vessel sign [36-38]. The bright vessel sign can also reveal certain distal arterial occlusions not initially detected on the vascular sequences, e.g. 3D TOF sequences [38].

\section{Post-therapeutic hyperperfusion}

When early arterial recanalisation occurs after intravenous thrombolysis, focal zones of hyperperfusion, termed luxury perfusion, can appear within the initial hypoperfusion zone. These zones are sometimes visible only on the ASL sequences and not on DSC-MRI, further complicating their interpretation [39-41].

Thus the presence of hyperperfusion zones on the ASL sequences of a control MRI early after thrombolysis is associated with improved functional outcome at $24 \mathrm{~h}$ and 3 months and with a smaller final infarct volume [39, 40]. These zones of hyperperfusion would correspond to preserved regions that achieve restitutio ad integrum after the acute episode [40] (Fig. 6).

In opposition, there is ongoing debate on how early hyperperfusion zones would be associated with haemorrhagic risk since the available evidence is contradictory [40, 41]. Nevertheless, outcome would be better in hyperperfusion patients independently of the presence or not of haemorrhagic transformation [40]. Post-therapeutic ASL perfusion status predicts outcome.

\section{Diagnosis of stroke mimics}

Stroke mimics are non-vascular neurological pathologies that reproduce the symptoms of stroke. According to the literature, they occur in 1 to $14.5 \%$ [42-48] of patients treated with intravenous thrombolysis for suspected acute stroke, with a mean of $4.38 \%$ [46]. These different studies report that these patients have a low risk of haemorrhage, estimated at 0 to $1 \%$,
Fig. 7 Brain MRI in a 65-yearold patient with sudden-onset left hemibody deficit. FLAIR (a), DWI (b and c), 3D TOF (d), ASL/ TOF fusion (e), and ASL (f) sequences in the axial plane. No visible lesion on the FLAIR sequence. $\mathbf{b}$ and $\mathbf{d}$ Hyperintense cortical zone on the DWI images showing a right temporo-parietooccipital zone not corresponding to a vascular territory (white arrow), with involvement of the homolateral pulvinar (arrowhead). $\mathbf{d}, \mathbf{e}$ and $\mathbf{f}$ Dilatation of the sylvian and right posterior cerebral arteries (parentheses) associated with elevated $\mathrm{CBF}$ (white arrows) in a context of status epilepticus

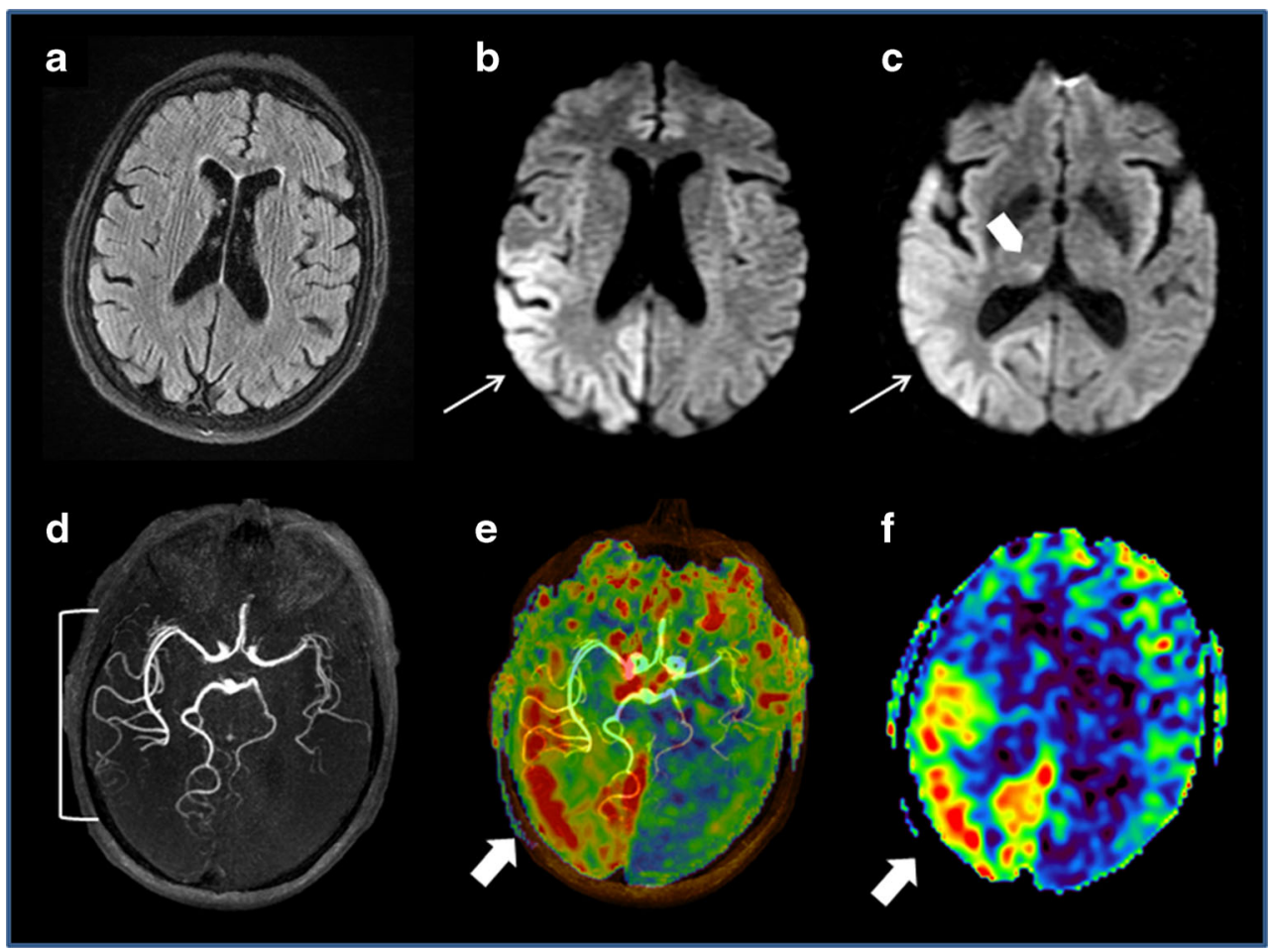


Fig. 8 Brain MRI in an 85-yearold patient with a history of right sylvian ischaemic stroke presenting with recurrent left hemibody deficiency. FLAIR (a and d), DWI (b and e), and ASL/DWI fusion ( $\mathbf{c}$ and $\mathbf{f}$ ) sequences in the axial plane. a, b, c Sequelar right posterior sylvian zone with no sign of recent ischaemia (white arrows). d, e, f Hyperperfusion zone bordering the superior part of the cavity (arrowhead), without FLAIR or DWI anomaly, related to a partial seizure on ischaemic sequelae
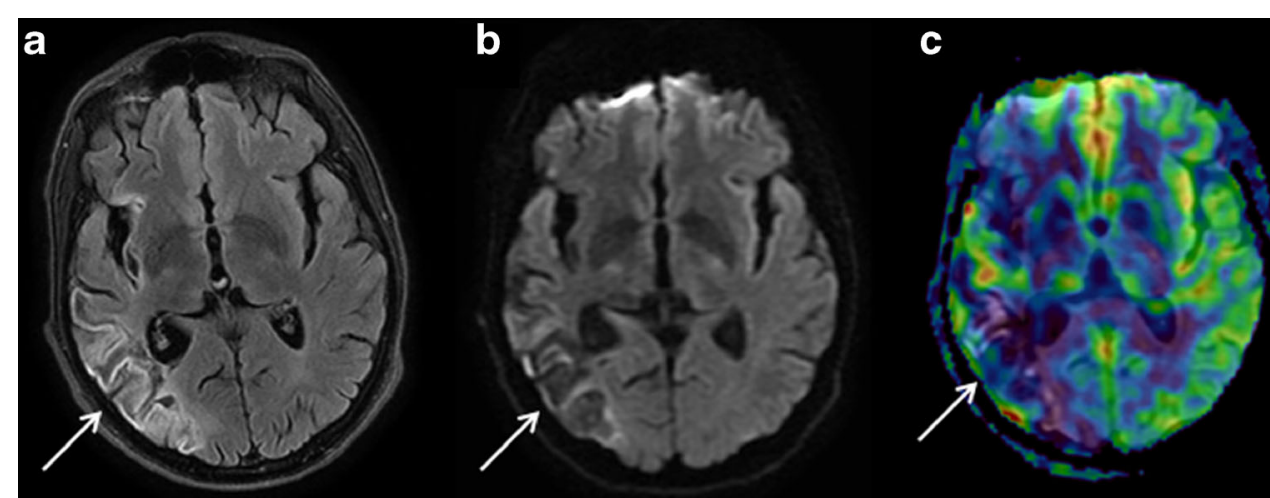

d

$\theta$

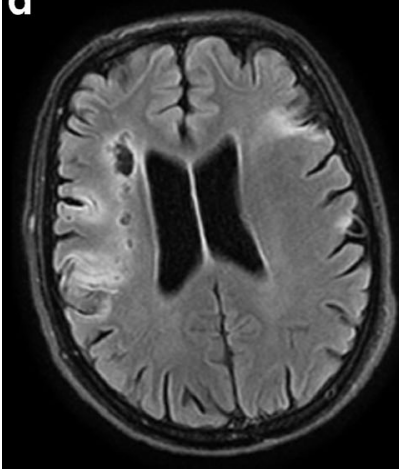

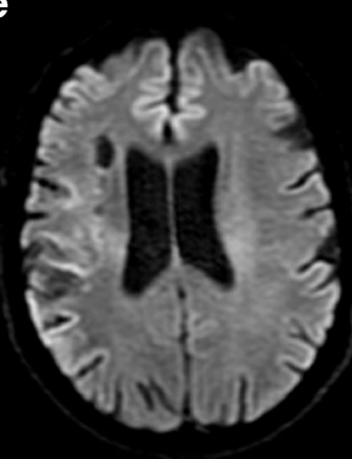

f

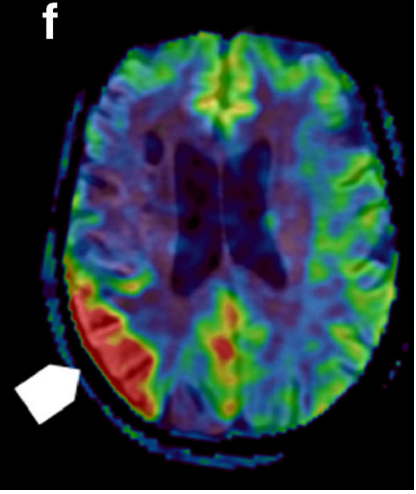

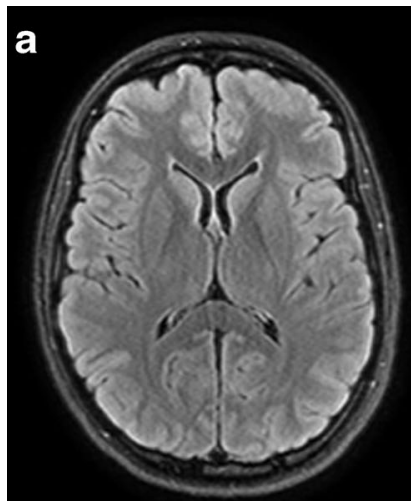

e

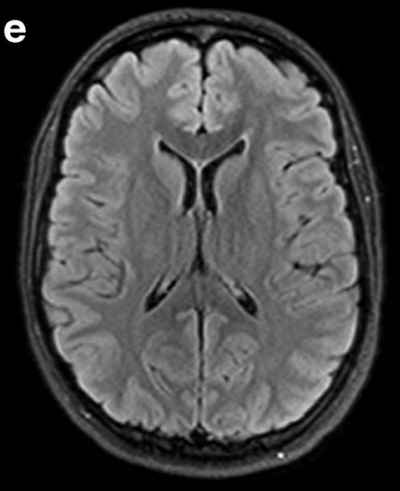

b

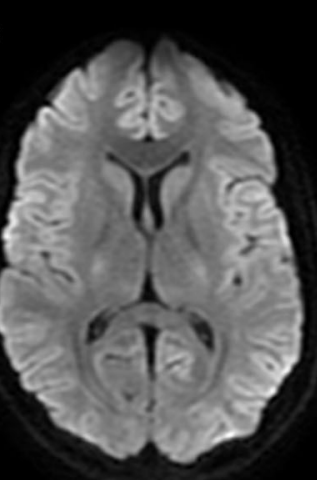

f

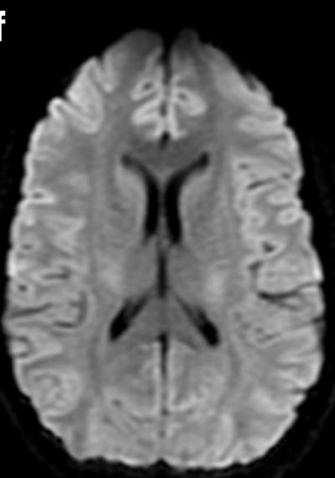

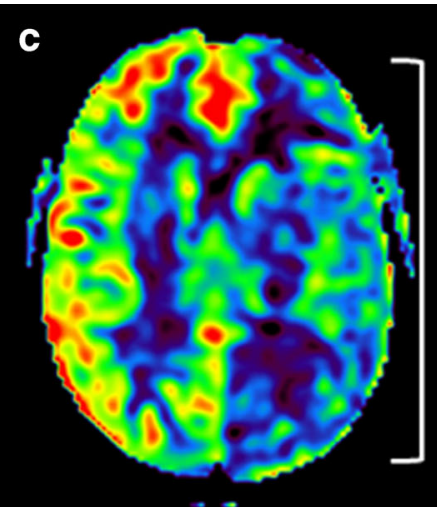

g

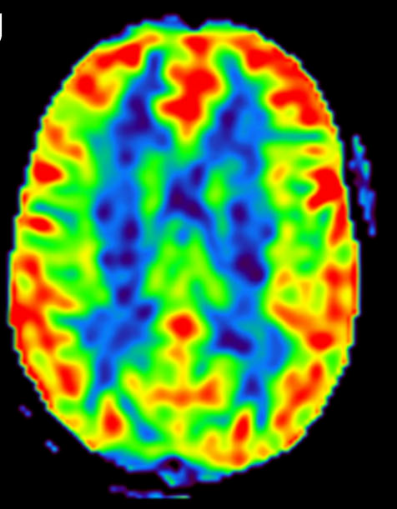

d

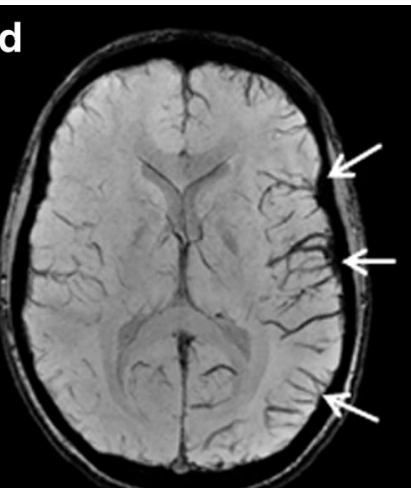

$\mathbf{h}$

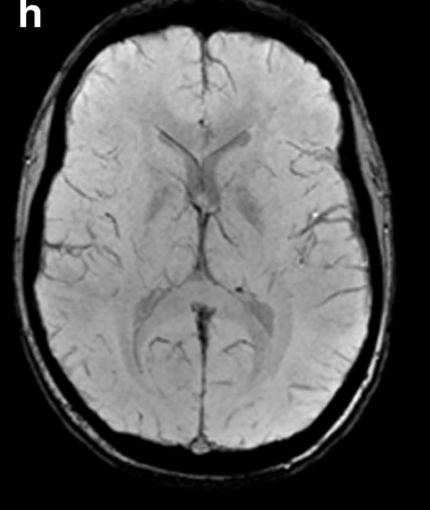

Fig. 9 A 21-year-old patient presenting sudden-onset aphasia. Initial (top row) and control (H24, bottom row) brain MRI with FLAIR (a, e), DWI $(\mathbf{b}, \mathbf{f}), \operatorname{ASL}(\mathbf{c}, \mathbf{g})$ and SWI (d, h) sequences in the axial plane. Initial MRI, $\mathbf{a}$ and $\mathbf{b}$ No visible acute ischaemic zone. $\mathbf{c}$ Large area of hypoperfusion

affecting the whole left hemisphere (parentheses) related to migraine aura. d Left hemispheric brush sign (white arrows). Control MRI (H24) shows no ischaemic lesion (e and $\mathbf{f}$ ), a normal left hemispheric perfusion (g), and a disappearance of the brush sign (h) 
and a better functional prognosis than patients who undergo thrombolysis for confirmed ischaemic stroke [49]. There is however a significant treatment-related cost increment, estimated at \$5400 in one American study [49]. These elements should incite efforts to optimise candidate selection for thrombolysis.

In order of frequency, the causes of stroke mimics are: partial epilepsy and psychiatric disorders, and then at variable frequencies depending on the report, infectious diseases (meningitis and meningoencephalitis), migraine with aura, brain tumours, cortical vein thrombosis, demyelinating inflammatory diseases, and metabolic or toxic pathologies. ASL can identify certain aetiologies of stroke mimics.

When there is an epileptic origin, ASL imaging shows focal hyperperfusion during the ictal and early post-ictal phases with increased CBF in the epileptogenic grey matter [50-52]. These zones of hyperperfusion are not limited to a single cerebral vessel territory and are frequently associated with suggestive morphological anomalies such as hypersignals from the pulvinars or the splenium of the corpus callosum on FLAIR and DWI sequences [53] (Fig. 7). ASL can also identify epileptogenic foci, which develop in ischaemic scar tissue in patients given emergency care for suspected recurrent stroke in a previous infarction zone, demonstrating high flow rate zones situated on the borders of parenchymatous sequelae (Fig. 8). In the inter-ictal phase, ASL can identify epileptogenic foci located in focal hypoperfusion zones [54].

In migraine aura, perfusion imaging can reveal anomalous focal brain perfusion with a long MTT and decreased CBF and cerebral blood volume $[55,56]$. In this situation ASL can also identify areas of decreased CBF [53]. These perfusion anomalies can sometimes resemble those observed in ischaemic stroke and may be associated with a brush sign [57] (Fig. 9), but are frequently bilateral, involving more than a single vascular territory, and predominating in posterior regions [53].

If the imaging is obtained late, at the headache phase, ASL reveals hyperperfusion with increased CBF $[53,58]$ that can be difficult to distinguish from other stroke mimics such as potential luxury perfusion. Nevertheless, the clinical presentation is usually sufficient to successfully guide diagnosis [53].

\section{Conclusion}

SWI and ASL sequences take advantage of the stronger magnetic field and have demonstrated their contribution to 3-T MRI exploration of acute ischaemic stroke. SWI provides prognostic elements useful for identifying the localisation, morphology, and aetiology of the thrombus, as well as the extent of the parenchymal hypoperfusion, while improving the spatial resolution and sensitivity of $\mathrm{T} 2$ gradient-echo sequences for the detection of haemorrhagic transformation. ASL should not be used as a routine sequence because of the acquisition time, but provides precious information for the differential diagnosis in specific situations, giving insight into the early post-therapeutic outcome and a noninvasive assessment of the penumbra.

\section{Compliance with ethical standards}

Conflict of interest None.

Funding None

Open Access This article is distributed under the terms of the Creative Commons Attribution 4.0 International License (http:// creativecommons.org/licenses/by/4.0/), which permits unrestricted use, distribution, and reproduction in any medium, provided you give appropriate credit to the original author(s) and the source, provide a link to the Creative Commons license, and indicate if changes were made.

\section{References}

1. Hodel J, Rodallec M, Gerber S, Blanc R, Maraval A, Caron S et al (2012) Susceptibility weighted magnetic resonance sequences "SWAN, SWI and VenoBOLD": technical aspects and clinical applications. J Neuroradiol J Neuroradiol 39(2):71-86

2. Wycliffe ND, Choe J, Holshouser B, Oyoyo UE, Haacke EM, Kido DK (2004) Reliability in detection of hemorrhage in acute stroke by a new three-dimensional gradient recalled echo susceptibilityweighted imaging technique compared to computed tomography: a retrospective study. J Magn Reson Imaging JMRI 20(3):372-377

3. Hayashida Y, Kakeda S, Hiai Y, Ide S, Ogasawara A, Ooki H et al (2014) Diagnosis of intracranial hemorrhagic lesions: comparison between 3D-SWAN (3D T2*-weighted imaging with multi-echo acquisition) and 2D-T2*-weighted imaging. Acta Radiol Stockh Swed 1987 55(2):201-207

4. Cheng A-L, Batool S, McCreary CR, Lauzon ML, Frayne R, Goyal $M$ et al (2013) Susceptibility-weighted imaging is more reliable than T2*-weighted gradient-recalled echo MRI for detecting microbleeds. Stroke J Cereb Circ 44(10):2782-2786

5. Nandigam RNK, Viswanathan A, Delgado P, Skehan ME, Smith EE, Rosand J et al (2009) MR imaging detection of cerebral microbleeds: effect of susceptibility-weighted imaging, section thickness, and field strength. AJNR Am J Neuroradiol 30(2):338343

6. Cho K-H, Kim JS, Kwon SU, Cho A-H, Kang D-W (2005) Significance of susceptibility vessel sign on $\mathrm{T} 2 *$-weighted gradient echo imaging for identification of stroke subtypes. Stroke J Cereb Circ 36(11):2379-2383

7. Kim HS, Lee DH, Choi CG, Kim SJ, Suh DC (2006) Progression of middle cerebral artery susceptibility sign on $\mathrm{T} 2 *$-weighted images: its effect on recanalization and clinical outcome after thrombolysis. AJR Am J Roentgenol 187(6):W650-W657

8. Kim SK, Yoon W, Kim TS, Kim HS, Heo TW, Park MS (2015) Histologic analysis of retrieved clots in acute ischemic stroke: correlation with stroke etiology and gradient-echo MRI. AJNR Am J Neuroradiol 36(9):1756-1762

9. Yamamoto N, Satomi J, Tada Y, Harada M, Izumi Y, Nagahiro S et al (2015) Two-layered susceptibility vessel sign on 3-Tesla T2*- 
weighted imaging is a predictive biomarker of stroke subtype. Stroke J Cereb Circ 46(1):269-271

10. Kimura K, Sakamoto Y, Aoki J, Iguchi Y, Shibazaki K, Inoue T (2011) Clinical and MRI predictors of no early recanalization within 1 hour after tissue-type plasminogen activator administration. Stroke J Cereb Circ 42(11):3150-3155

11. Kimura K, Iguchi Y, Shibazaki K, Watanabe M, Iwanaga T, Aoki J (2009) M1 susceptibility vessel sign on T2* as a strong predictor for no early recanalization after IV-t-PA in acute ischemic stroke. Stroke J Cereb Circ 40(9):3130-3132

12. Aoki J, Kimura K, Shibazaki K, Sakamoto Y, Saji N, Uemura J (2013) Location of the susceptibility vessel sign on T2*-weighted MRI and early recanalization within 1 hour after tissue plasminogen activator administration. Cerebrovasc Dis Extra 3(1):111-120

13. Yan S, Hu H, Shi Z, Zhang X, Zhang S, Liebeskind DS et al (2014) Morphology of susceptibility vessel sign predicts middle cerebral artery recanalization after intravenous thrombolysis. Stroke J Cereb Circ 45(9):2795-2797

14. Yan S, Chen Q, Zhang X, Xu M, Han Q, Shao A et al (2015) Extensive blooming artifact predicts no recanalization after intravenous thrombolysis. Eur J Neurol 26

15. Bourcier R, Volpi S, Guyomarch B, Daumas-Duport B, LintiaGaultier A, Papagiannaki C et al (2015) Susceptibility vessel sign on MRI predicts favorable clinical outcome in patients with anterior circulation acute stroke treated with mechanical thrombectomy. AJNR Am J Neuroradiol 36(12):2346-2353

16. Soize S, Batista AL, Rodriguez Regent C, Trystram D, Tisserand M, Turc G et al (2015) Susceptibility vessel sign on T2* magnetic resonance imaging and recanalization results of mechanical thrombectomy with stent retrievers: a multicentre cohort study. Eur J Neurol 22(6):967-972

17. Allibert R, Billon Grand C, Vuillier F, Cattin F, Muzard E, Biondi A et al (2014) Advantages of susceptibility-weighted magnetic resonance sequences in the visualization of intravascular thrombi in acute ischemic stroke. Int J Stroke Off J Int Stroke Soc 9(8):980 984

18. Hodel J, Leclerc X, Khaled W, Tamazyan R, Rodallec M, Gerber S et al (2014) Comparison of 3D multi-echo gradient-echo and 2D T2* MR sequences for the detection of arterial thrombus in patients with acute stroke. Eur Radiol 24(3):762-769

19. Park M-G, Yoon CH, Baik SK, Park K-P (2015) Susceptibility vessel sign for intra-arterial thrombus in acute posterior cerebral artery infarction. J Stroke Cerebrovasc Dis Off J Natl Stroke Assoc 24(6):1229-1234

20. Park M-G, Oh S-J, Baik SK, Jung DS, Park K-P (2016) Susceptibility-weighted imaging for detection of thrombus in acute cardioembolic stroke. J Stroke 18(1):73-79

21. Radbruch A, Mucke J, Schweser F, Deistung A, Ringleb PA, Ziener $\mathrm{CH}$ et al (2013) Comparison of susceptibility weighted imaging and TOF-angiography for the detection of thrombi in acute stroke. PLoS One 8(5), e63459

22. Gratz PP, Schroth G, Gralla J, Mattle HP, Fischer U, Jung S et al (2015) Whole-brain susceptibility-weighted thrombus imaging in stroke: fragmented thrombi predict worse outcome. AJNR Am J Neuroradiol 36(7):1277-1282

23. Patzig M, Feddersen B, Haegler K, Olzowy B, Mees K, Fischer R et al (2015) Susceptibility-weighted angiography visualizes hypoxia in cerebral veins. Invest Radiol 50(6):397-400

24. Chalian M, Tekes A, Meoded A, Poretti A, Huisman TAGM (2011) Susceptibility-weighted imaging (SWI): a potential non-invasive imaging tool for characterizing ischemic brain injury? J Neuroradiol 38(3):187-190

25. Park M-G, Yang T-I, Oh S-J, Baik SK, Kang YH, Park K-P (2014) Multiple hypointense vessels on susceptibility-weighted imaging in acute ischemic stroke: surrogate marker of oxygen extraction fraction in penumbra? Cerebrovasc Dis Basel Switz 38(4):254-261
26. Chen C-Y, Chen C-I, Tsai FY, Tsai P-H, Chan WP (2015) Prominent vessel sign on susceptibility-weighted imaging in acute stroke: prediction of infarct growth and clinical outcome. PLoS One 10(6):e0131118

27. Luo S, Yang L, Wang L (2015) Comparison of susceptibilityweighted and perfusion-weighted magnetic resonance imaging in the detection of penumbra in acute ischemic stroke. J Neuroradiol 42(5):255-260

28. Terasawa Y, Yamamoto N, Morigaki R, Fujita K, Izumi Y, Satomi J et al (2014) Brush sign on 3-T T2*-weighted MRI as a potential predictor of hemorrhagic transformation after tissue plasminogen activator therapy. Stroke J Cereb Circ 45(1):274-276

29. Alsop DC, Detre JA, Golay X, Günther M, Hendrikse J, Hernandez-Garcia L et al (2015) Recommended implementation of arterial spin-labeled perfusion MRI for clinical applications: a consensus of the ISMRM perfusion study group and the European consortium for ASL in dementia. Magn Reson Med 73(1):102-116

30. Bivard A, Krishnamurthy V, Stanwell P, Levi C, Spratt NJ, Davis S et al (2014) Arterial spin labeling versus bolus-tracking perfusion in hyperacute stroke. Stroke J Cereb Circ 45(1):127-133

31. Bokkers RPH, Hernandez DA, Merino JG, Mirasol RV, van Osch MJ, Hendrikse J et al (2012) Whole-brain arterial spin labeling perfusion MRI in patients with acute stroke. Stroke J Cereb Circ 43(5):1290-1294

32. Mirasol RV, Bokkers RPH, Hernandez DA, Merino JG, Luby M, Warach S et al (2014) Assessing reperfusion with whole-brain arterial spin labeling: a noninvasive alternative to gadolinium. Stroke J Cereb Circ 45(2):456-461

33. Wang DJJ, Alger JR, Qiao JX, Hao Q, Hou S, Fiaz R et al (2012) The value of arterial spin-labeled perfusion imaging in acute ischemic stroke: comparison with dynamic susceptibility contrastenhanced MRI. Stroke J Cereb Circ 43(4):1018-1024

34. Niibo T, Ohta H, Yonenaga K, Ikushima I, Miyata S, Takeshima H (2013) Arterial spin-labeled perfusion imaging to predict mismatch in acute ischemic stroke. Stroke J Cereb Circ 44(9):2601-2603

35. Wang J, Alsop DC, Li L, Listerud J, Gonzalez-At JB, Schnall MD et al (2002) Comparison of quantitative perfusion imaging using arterial spin labeling at 1.5 and 4.0 Tesla. Magn Reson Med 48(2): 242-254

36. Majer M, Mejdoubi M, Schertz M, Colombani S, Arrigo A (2015) Raw arterial spin labeling data can help identify arterial occlusion in acute ischemic stroke. Stroke J Cereb Circ 46(6):e141-e144

37. Tada Y, Satomi J, Abe T, Kuwayama K, Sogabe S, Fujita K et al (2014) Intra-arterial signal on arterial spin labeling perfusion MRI to identify the presence of acute middle cerebral artery occlusion. Cerebrovasc Dis Basel Switz 38(3):191-196

38. Yoo R-E, Yun TJ, Rhim JH, Yoon B-W, Kang KM, Choi SH et al (2015) Bright vessel appearance on arterial spin labeling MRI for localizing arterial occlusion in acute ischemic stroke. Stroke J Cereb Circ 46(2):564-567

39. Bivard A, Stanwell P, Levi C, Parsons M (2013) Arterial spin labeling identifies tissue salvage and good clinical recovery after acute ischemic stroke. J Neuroimaging Off $\mathrm{J}$ Am Soc Neuroimaging 23(3):391-396

40. Viallon M, Altrichter S, Pereira VM, Nguyen D, Sekoranja L, Federspiel A et al (2010) Combined use of pulsed arterial spinlabeling and susceptibility-weighted imaging in stroke at 3T. Eur Neurol 64(5):286-296

41. Yu S, Liebeskind DS, Dua S, Wilhalme H, Elashoff D, Qiao XJ et al (2015) Postischemic hyperperfusion on arterial spin labeled perfusion MRI is linked to hemorrhagic transformation in stroke. J Cereb Blood Flow Metab Off J Int Soc Cereb Blood Flow Metab 35(4):630-637

42. Artto V, Putaala J, Strbian D, Meretoja A, Piironen K, Liebkind R et al (2012) Stroke mimics and intravenous thrombolysis. Ann Emerg Med 59(1):27-32 
43. Förster A, Griebe M, Wolf ME, Szabo K, Hennerici MG, Kern R (2012) How to identify stroke mimics in patients eligible for intravenous thrombolysis? J Neurol 259(7):1347-1353

44. Guillan M, Alonso-Canovas A, Gonzalez-Valcarcel J, Garcia Barragan N, Garcia Caldentey J, Hernandez-Medrano I et al (2012) Stroke mimics treated with thrombolysis: further evidence on safety and distinctive clinical features. Cerebrovasc Dis Basel Switz 34(2):115-120

45. Lewandowski C, Mays-Wilson K, Miller J, Penstone P, Miller DJ, Bakoulas K et al (2015) Safety and outcomes in stroke mimics after intravenous tissue plasminogen activator administration: a singlecenter experience. J Stroke Cerebrovasc Dis Off J Natl Stroke Assoc 24(1):48-52

46. Tsivgoulis G, Zand R, Katsanos AH, Goyal N, Uchino K, Chang J et al (2015) Safety of intravenous thrombolysis in stroke mimics: prospective 5-year study and comprehensive meta-analysis. Stroke J Cereb Circ 46(5):1281-1287

47. Winkler DT, Fluri F, Fuhr P, Wetzel SG, Lyrer PA, Ruegg S et al (2009) Thrombolysis in stroke mimics: frequency, clinical characteristics, and outcome. Stroke J Cereb Circ 40(4):1522-1525

48. Zinkstok SM, Engelter ST, Gensicke H, Lyrer PA, Ringleb PA, Artto V et al (2013) Safety of thrombolysis in stroke mimics: results from a multicenter cohort study. Stroke J Cereb Circ 44(4):1080-1084

49. Goyal N, Male S, Al Wafai A, Bellamkonda S, Zand R (2015) Cost burden of stroke mimics and transient ischemic attack after intravenous tissue plasminogen activator treatment. J Stroke Cerebrovasc Dis Off J Natl Stroke Assoc 24(4):828-833

50. Altrichter S, Pendse N, Wissmeyer M, Jägersberg M, Federspiel A, Viallon M et al (2009) Arterial spin-labeling demonstrates ictal cortical hyperperfusion in epilepsy secondary to hemimegalencephaly. J Neuroradiol J Neuroradiol 36(5):303-305

51. Deibler AR, Pollock JM, Kraft RA, Tan H, Burdette JH, Maldjian JA (2008) Arterial spin-labeling in routine clinical practice, part 3: hyperperfusion patterns. AJNR Am J Neuroradiol 29(8):1428-1435

52. Oishi M, Ishida G, Morii K, Hasegawa K, Sato M, Fujii Y (2012) Ictal focal hyperperfusion demonstrated by arterial spin-labeling perfusion MRI in partial epilepsy status. Neuroradiology 54(6): 653-656

53. Danière F, Edjlali-Goujon M, Mellerio C, Turc G, Naggara O, Tselikas L et al (2014) MR screening of candidates for thrombolysis: how to identify stroke mimics? J Neuroradiol 41(5):283-295

54. Pendse N, Wissmeyer M, Altrichter S, Vargas M, Delavelle J, Viallon $M$ et al (2010) Interictal arterial spin-labeling MRI perfusion in intractable epilepsy. J Neuroradiol J Neuroradiol 37(1):60-63

55. Floery D, Vosko MR, Fellner FA, Fellner C, Ginthoer C, Gruber F et al (2012) Acute-onset migrainous aura mimicking acute stroke: MR perfusion imaging features. AJNR Am J Neuroradiol 33(8): $1546-1552$

56. Förster A, Wenz H, Kerl HU, Brockmann MA, Groden C (2014) Perfusion patterns in migraine with aura. Cephalalgia Int $\mathrm{J}$ Headache 34(11):870-876

57. Karaarslan E, Uluus S, Kürtüncü M (2001) Susceptibility-weighted Imaging in migraine with aura. AJNR Am J Neuroradiol 32(1):E5-E7

58. Pollock JM, Deibler AR, Burdette JH, Kraft RA, Tan H, Evans AB et al (2008) Migraine associated cerebral hyperperfusion with arterial spin-labeled MR imaging. AJNR Am J Neuroradiol 29(8): 1494-1497 